\title{
Review of dietary approaches for treatment of gestational diabetes mellitus
}

\author{
Clare Viglione* and Jagriti Upadhyay \\ Dimock Community Health Center, Boston, MA, USA
}

\begin{abstract}
Gestational Diabetes Mellitus (GDM) is the most common medical disorder during pregnancy and has severe and long-lasting implications both for the mother and child. Nearly $10 \%$ of all United States pregnant females develop GDM.Dietary therapy is first line treatment and should be initiated as soon as GDM is diagnosed, however, consensus on specific dietary recommendations has not yet been achieved and nutrition counseling may vary widely from practice to practice. This systematic literature review encompasses novel supplement-based treatments (e.g. supplementation of fatty acids, vitamins, and minerals) and traditional diets (e.g. Glycemic Index Diet or Low Carbohydrate Diet) as well as evaluates the evidence for their application.
\end{abstract}

\section{Introduction}

Gestational Diabetes Mellitus (GDM) is a common condition during pregnancy but has severe and long-lasting implications both for the mother and child. Gestational Diabetes develops during pregnancy as an exacerbation of a naturally occurring insulin-resistant state. It is defined as any degree of glucose intolerance with onset or first recognition during pregnancy [1]. GDM is the most common type of diabetes during pregnancy, as $90 \%$ of pregnant women with diabetes have GDM as opposed to Type 1 or Type 2 Diabetes [2].

Although the exact physiological mechanism of GDM has not been elucidated, potential contributing factors include increased plasma volume in early gestation, increased glucose consumption due to increased feto-placental glucose uptake in late gestation, impaired pancreatic beta cell function, and increased hepatic glucose production [3]. Pregnant women and those with GDM have a decrease in their mean fasting plasma glucose (FPG) level, but their hepatic glucose production is greatly increased [4]. This is coupled with decreases in insulin sensitivity to approximately one third of normal insulin sensitivity in a non-pregnant state [4].

Hyperglycemia directly impacts fetal health. Since glucose crosses the placenta by facilitated diffusion, fetuses from mothers with GDM stimulate islet cell proliferation and insulin production leading to macrosomia. Attention to maternal weight gain is important in minimizing the risk of fetal macrosomia. Maternal obesity, excess gestational weight gain, and GDM are independent and additive risk factors for macrosomia. GDM also has long lasting effects on the mother's health. GDM predisposes a woman to diabetes, metabolic syndrome and obesity in later life. Of all risk factors, GDM is the leading, most reliable predictor of Type II Diabetes in women.

Prevalence estimates for GDM vary widely. The Center for Disease Control for Disease Control reports that $9.2 \%$ of all United States pregnant females develop GDM [5]. Concomitant with the rise in obesity globally, prevalence of GDM is increasing both in high-income as well as low- and middle-income countries [6]. Maternal age of pregnancy and BMI are also increasing internationally which elevate the risk of developing GDM [7]. Universal screening for GDM may also contribute to a rise in prevalence. In 2014, the U.S. Preventive Services Task Force updated its 2008 statement to recommend screening of all asymptomatic healthy women at 24 weeks gestation (and earlier for those with known risk factors) [8]. Up to 50\% of women with GDM have none of the traditional risk factors (e.g., obesity, advanced maternal age, family history of diabetes, short stature, etc.) which supports universal screening to capture those without salient risk factors [9].

\section{Current dietary management of GDM}

The role of Medical Nutrition Therapy as a modifiable factor in the management of GDM is well accepted. However, consensus on specific dietary recommendations has not been achieved and thus diet therapy may vary widely from practice to practice. Guidelines on diet and lifestyle recommendations are similarly vague between leading professional organizations, Academy of Nutrition Dietetics, American College of Obstetrics and Gynecology, and American Diabetes Association, but do agree that dietary therapy should be initiated as soon as GDM is diagnosed, and insulin and other pharmacologic treatments should only be offered if patient cannot manage hyperglycemia with behavioral changes alone [10-20]. Insulin is not readily accepted by pregnant women and is inconvenient, expensive and a source of anxiety [21]. Generally a diet that aligns with the DRI guidelines for pregnancy is recommended, suggesting that $175 \mathrm{~g}$ of carbohydrate be provided daily for the developing fetus along with adequate energy and protein.

\section{Purpose of systematic literature review}

The purpose of this systematic literature review is to identify both existing and novel dietary treatments of GDM and evaluate the evidence for their application. The review encompasses supplementbased treatments (e.g., supplementation of fatty acids, vitamins, and

${ }^{\star}$ Correspondence to: Clare Viglione, USA, E-mail: cviglione@health.ucsd.edu

Received: September 07, 2020; Accepted: October 13, 2020; Published: October 16, 2020 
minerals) as well as traditional diets (e.g., Glycemic Index Diet or Low Carbohydrate Diet).

\section{Methods}

A review of the literature was conducted using PubMed and Google Scholar. Trials were evaluated based on predetermined inclusion and exclusion criteria listed in Table 1. Search terms were entered in a format acceptable to the PubMed search engine (i.e. diabetes, gestational [Mesh] OR gestational diabet ${ }^{\star}[$ tiab] OR pregnancy induced diabetes [tiab]) AND random $\left.{ }^{\star}[\mathrm{tw}]\right)$. Articles published between the years of 1998 and 2019 were included. A reverse search of citation references was completed. Studies were excluded if they involved nonhuman subjects and if the manuscript was not available in English. Studies were included if they were randomized controlled trials (RCTs) and excluded if they were non-randomized, observational, or crosssectional analyses.

To simplify comparison between clinical trials, randomized controlled trials were organized in tabular format (Table 2) and outcomes of mean change in fasting plasma glucose $(\mathrm{mg} / \mathrm{dL})$, mean change in fasting insulin $(\mathrm{mIU} / \mathrm{mL})$, and commencement of insulin therapy (categorical: $\mathrm{Y} / \mathrm{N}$ ) were delineated. These outcomes were selected given a likelihood of reporting in clinical trials. Moreover, the most commonly used marker of glycemia, glycated hemoglobin (HbAlc), is an unreliable marker of mean plasma glucose during pregnancy given higher than average red blood cell turnover increasing odds of false negative results [22-25]. In a sample of women with GDM, fasting glucose was the strongest predictor for large for gestational age infants which is associated with infant respiratory distress syndrome, various birth injuries and polycythemia [24].

\section{Results}

\section{Diet: Low carbohydrate}

Given that carbohydrate consumption is consistently and positively associated with postprandial glycemic rise, reducing dietary carbohydrate to control glucose has been the target of many dietary interventions [26]. In observational studies, carbohydrate restriction is associated with improved glycemic control, insulin sensitivity, and reduced need for insulin therapy [27]. However in this literature review, only one RCT of low carbohydrate ( $40 \%$ carbohydrate) diet versus control ( $\sim 5 \%$ carbohydrate) met criteria and did not demonstrate any statistically significant effect on plasma glucose or plasma insulin (Table 3) [28].

\section{Diet: Low glycemic index}

A glycemic index (GI) system was developed based on the notion that certain carbohydrate-containing foods are more likely to cause postprandial increases in plasma glucose. The GI is a standardized system which assigns numerical values to foods based on their propensity to increase blood glucose [29]. A low GI diet encourages the consumption of foods with a low GI score to reduce the impact of carbohydrate on glycemia, thereby managing diabetes and cardiovascular risk [29]. Six RCTs were selected for inclusion in this review (Table 3) [30-36]. Two out of 4 of the RCTs found significant differences in plasma blood glucose after the intervention and 2 out of 4 of the RCTs found significant differences in commencement of insulin therapy post-intervention. One out of 4 RCTs did not find statistically significant differences in mean plasma blood glucose change.

\section{Diet: High PUFA}

Patients with diabetes tend to have a higher intake of saturated fat as compared with polyunsaturated fatty acids (PUFA) [37]. Increasing dietary intake of polyunsaturated fatty acids is posited to enhance glucose transporter mRNA expression thereby enhancing hypoglycemic effects of insulin which should ultimately improve glucose control $[38,39]$. One RCT tested a high PUFA diet heavily enriched with sunflower oil versus a low PUFA diet in women with diagnosed GDM and found no significant difference in mean change plasma blood glucose or mean insulin change after the intervention (Table 3) [40].

\section{Diet: High MUFA}

MUFA specifically, may play a role in glycemic control because of anti-inflammatory effects on insulin sensitivity. MUFA intake is also associated with reduced risk of metabolic syndrome [41]. However only one randomized trial was identified which did not demonstrate an effect of high MUFA diet for five weeks on glycemic markers in patients with GDM (Table 3) [42].

\section{Diet: Dietary approach to stop hypertension (DASH)}

The DASH diet was originally developed for patients with hypertension, however DASH is high in fruits and vegetables, contains lean protein sources like chicken, fish and beans and low fat dairy products, and also low in red meat, salt, added sugars, and fat which should theoretically improve glucose and insulin sensitivity [43]. The DASH diet is also inherently low-glycemic, low energy-density and contains high amounts of fiber, phytoestrogens, potassium, magnesium, and folate that are helpful in reducing cardiovascular risk and metabolic syndrome [43]. Azadbakht et al. have demonstrated that consumption of the DASH diet for 8 weeks along with exercise was associated with improvements in insulin sensitivity in overweight patients without diabetes [44]. In this review, two RCTs, conducted by Asemi and coauthors, met inclusion and exclusion criteria. The first RCT demonstrated a statistically significant decrease in mean plasma blood glucose ( -7.62 vs. $3.68 ; \mathrm{p}=0.02)$ and insulin $(-2.62$ vs. $4.32 ; \mathrm{p}=0.03)$ and the second study demonstrated a significant reduction in number of patients to commence insulin therapy $(23 \%$ vs. $73 \%$; $<<0.0001)$ in patients with GDM after they adhered to DASH for 4 weeks (Table 3) $[44,45]$.

\section{Diet: Low Calorie}

Energy restriction is critical to the management of overweight and obesity, often comorbid with gestational diabetes. Rae et al. [71] tested caloric restriction versus a standard caloric diet in 120 Australian women with both obesity and gestational diabetes. Women in the intervention group were counseled to consume a diet that provided 70 percent of the calories recommended by the National Health and Medical Research Council of Australia (i.e. between 1590 and 1776 kilocalories per day) whereas those in the control group were counseled to consume 100 percent of the recommended calories (i.e. between 2010 and 2220 kilocalories per day) in the control group. There were no statistically significant differences in fasting plasma glucose or percentage of women who commenced insulin treatment. In addition, there were no adverse effects on pregnancy outcomes by consuming $30 \%$ fewer kilocalories than the recommended amount.

\section{Diet: High complex carbohydrate and low fat}

Complex carbohydrates which includes starchy vegetables like sweet potatoes or legumes tend to blunt postprandial glucose spikes 
as compared with simple carbohydrate. Additionally, reducing dietary fat, especially saturated fat, may increase the activity of cellular glucose transporters contributing to improved glycemic control. We identified two small RCTs testing a high complex carbohydrate (low fat) diet against a low carbohydrate (high fat) diet in patients with GDM. Neither study demonstrated any statistically significant differences in insulin levels, however in one of the two trials the authors did note that fasting glucose decreased with the complex carbohydrate diet, however statistics were not reported $[46,47]$.

\section{Supplement: Vitamin D}

The effect of vitamin D supplementation on diabetes might be mediated by an effect on apolipoprotein gene expression and inflammation [48]. A systematic review and meta-analysis of vitamin $\mathrm{D}$ in adults with T2DM found a modest reduction of HbA1C after treatment, but no difference in fasting blood glucose [49]. However, a 6 month clinical trial of 4,000 IU cholecalciferol daily resulted in a significant increase in insulin sensitivity in those that were vitamin D deficient at baseline [50]. Two RCTs were identified assessing vitamin $\mathrm{D}$ in the gestational diabetes population. Asemi et al. tested the effect of 2 doses of 50,000 IU at baseline and at 3 weeks which resulted in statistically significant reductions in fasting plasma glucose and fasting insulin [51,52].

\section{Supplement: Omega 3-fatty acid}

Epidemiologic evidence suggests a correlation between GDM and reduced levels of essential omega-3 fatty acids as compared with women without GDM [53]. Additionally, researchers have demonstrated that supplementation with omega-3 fats increases peripheral glucose uptake and utilization in animals [54]. A systematic review of the evidence for omega-3 fatty acid supplementation in patients with GDM found only one rigorous randomized trial, a small study $(n=56)$ in an Iranian population [55]. The trial did see a statistically significant effect of supplementation with omega-3 containing $180 \mathrm{mg}$ of EPA and $120 \mathrm{mg}$ of DHA on fasting insulin (Table 3) [55].

\section{Supplement: Selenium}

A systematic review of six observational studies demonstrated that serum selenium is significantly lower in pregnant women with hyperglycemia compared to pregnant women without hyperglycemia [56]. Moreover, selenium supplementation has been shown to improve markers of glucose homeostasis, inflammation and oxidative stress in healthy populations [57]. Selenium may also inhibit the production of advanced glycation end products and free radicals which contribute to peripheral insulin resistance [58]. The availability of rigorous RCTs testing selenium supplementation on glycemia in patients with GDM are limited. Asemi et al. (Table 3) did find that six weeks of supplementation resulted in significant reductions in fasting plasma glucose and insulin [57].

\section{Supplement: Probiotics}

Probiotics may attenuate glucose absorption from the gut and, in some studies, have led to improvements in glucose tolerance in pregnancy [58]. We identified six RCTs testing the use of probiotics in patients with GDM and 1 RCT testing the effect of synbiotics, a probiotic capsule with fructose saccharide fiber hypothesized to promote bacterial growth [59]. In one trial, Dolatkhah et al. evaluated the impact of a daily probiotic (with 4 common bacterial strains) on fasting blood glucose and fasting insulin in a patient population with GDM. They found that probiotic supplementation led to a marked decrease in mean fasting blood glucose $(-15.3 \mathrm{mg} / \mathrm{dL}$ vs. $-7.3 \mathrm{mg} / \mathrm{dL})$ in six weeks [60]. They did not however see a difference in fasting insulin. Lindsay et al. on the other hand conducted a small RCT testing a probiotic with one bacterial strain on patients with GDM for 4-6 weeks and found no difference in glucose or insulin $[61,62]$. In a more recent study of a 2-strain probiotic supplement in 58 women with GDM, supplementation led to smaller increases in fasting plasma glucose and insulin as compared with those that received placebo ( 0.68 vs. 4.62 , $\mathrm{p}=0.03$ vs. 1.11 vs $3.77, \mathrm{p}=0.01$, respectively) [73].

\section{Supplement: Oral protein hydrolysate}

Protein supplements have been reported to reduce postprandial glucose rise as protein typically takes longer to digest than carbohydrates. Studies have confirmed that pre-meal consumption of whey protein reduces postprandial blood glucose. In this review, only one RCT was identified in the GDM population testing the effects of a protein hydrolysate drink before breakfast and dinner. After 7 days of pre-meal protein, there was no statistically significant effect on FPG or fasting insulin however post-prandial glucose did reduce by $5-10 \%$ in the treatment arm [74].

\section{Supplement: Magnesium}

Type 2 diabetes is associated with intracellular and extracellular deficiencies in magnesium and a high prevalence of magnesium deficits have been identified in those with poorly controlled hyperglycemia [63]. Pregnancy is also independently associated with magnesium depletion and GDM can exacerbate this trend [64]. Current data have suggested a positive effect of magnesium supplementation on the metabolic status of healthy pregnant women [64]. Asemi et al. assessed the effect of $250 \mathrm{mg}$ magnesium oxide vs placebo for six weeks on 70 pregnant women with GDM (Table 3) and found statistically significant differences in mean FPG change ( -9.7 vs. $+1.8 \mathrm{mg} / \mathrm{dL})$ and insulin $(-2.1 \mathrm{vs.}+5.7 \mathrm{mIU} / \mathrm{mL}$; Table 3) [65]. Moreover, magnesium and vitamin E co-supplementation (i.e. $250 \mathrm{mg}$ magnesium oxide plus $400 \mathrm{IU} /$ day vitamin $\mathrm{E}$ vs. placebo) for six weeks in 60 women with GDM resulted in mean reductions in fasting plasma glucose ( -4.9 vs. -0.1 ; no p-value) and insulin ( -1.7 vs. +1.5 ; no p-value) [75].

\section{Supplements: Myoinositol}

Inositol, or more specifically Myoinositol, is one of the intracellular mediators of the insulin signaling pathway and has been purported to improve management of diabetes, PCOS, and metabolic syndrome. It is posited that myoinositol as a dietary supplement may improve

Table 1. Inclusion criteria for dietary studies

\begin{tabular}{|l|l|}
\hline \multicolumn{1}{|c|}{ Inclusion } & \multicolumn{1}{|c|}{ Exclusion } \\
\hline $\begin{array}{l}\text { Randomized-controlled trials assessing } \\
\text { dietary intervention on pregnant females } \\
\text { with diagnosed gestational diabetes or } \\
\text { hyperglycemia }\end{array}$ & $\begin{array}{l}\text { Non-randomized and non-controlled; } \\
\text { Observational or Longitudinal }\end{array}$ \\
\hline $\begin{array}{l}\text { Inclusionary terms: diabetes, } \\
\text { gestational[Mesh] OR gestational } \\
\text { diabet*[tiab] OR pregnancy induced } \\
\text { diabetes[tiab]) AND random*[tw] }\end{array}$ & Not available in English \\
\hline Published between 1999-2019 & $\begin{array}{l}\text { Long term outcomes (i.e. birth outcomes, } \\
\text { maternal outcomes post-pregnancy or child } \\
\text { outcomes); Outcomes not available }\end{array}$ \\
\hline Diet-controlled GDM & $\begin{array}{l}\text { Study population includes those with Type } \\
1 \text { or Type 2 Diabetes }\end{array}$ \\
\hline & $\begin{array}{l}\text { Medication-controlled or Insulin-controlled } \\
\text { GDM }\end{array}$ \\
\hline & $\begin{array}{l}\text { Exclusionary terms [title]: safety; } \\
\text { tolerability; exercise, physical activity }\end{array}$ \\
\hline
\end{tabular}


Table 2. Summary of Randomized Trials assessing supplement-based treatments of GDM

\begin{tabular}{|c|c|c|c|c|c|c|c|}
\hline Supplement & Author; Year & Design & $\mathbf{n}$ & Independent Variable & $\begin{array}{l}\text { Mean change in } \\
\text { FPG }(\mathrm{mg} / \mathrm{dL})\end{array}$ & $\begin{array}{l}\text { Mean change in } \\
\text { serum insulin } \\
(\mathrm{mIU} / \mathrm{mL})\end{array}$ & $\begin{array}{c}\text { Initiation } \\
\text { of Insulin } \\
(\%)\end{array}$ \\
\hline $\begin{array}{l}\text { Oral protein } \\
\text { hydrolysate }\end{array}$ & Saleh et al. 2018 & $\mathrm{RCT}$ & $\mathrm{n}=50$ & $\begin{array}{c}\text { A protein hydrolysate drink, } 8.5 \mathrm{~g} \\
\text { before breakfast and } 8.5 \mathrm{~g} \text { before } \\
\text { dinner vs. placebo drink (no energy } \\
\text { content) }\end{array}$ & $\begin{array}{l}-0.01 \text { vs. } 0.00 \\
\text { No p-value }\end{array}$ & $\begin{array}{l}-13.0 \text { vs. }-1.0 \\
\text { No p-value }\end{array}$ & --- \\
\hline $\begin{array}{l}\text { Omega } 3 \text {-fatty } \\
\text { acid }\end{array}$ & $\begin{array}{l}\text { Samimi et al. } \\
2015\end{array}$ & $\begin{array}{l}\text { RCT; double } \\
\text { blind; placebo- } \\
\text { controlled }\end{array}$ & $\mathrm{n}=56$ & $\begin{array}{l}\text { 1000mg omega-3-fatty acid } \\
\text { (180mg EPA; } 120 \mathrm{mg} \text { DHA) daily } \\
\text { for six weeks vs. placebo }\end{array}$ & $\begin{array}{l}0.9 \text { vs. } 5.6 \\
\mathrm{p}=0.12\end{array}$ & $\begin{array}{c}-1.5 \text { vs. } 3.5 \\
p=0.02\end{array}$ & --- \\
\hline Magnesium & $\begin{array}{l}\text { Asemi et al. } \\
\quad 2015\end{array}$ & $\begin{array}{l}\text { RCT; double } \\
\text { blind; placebo- } \\
\text { controlled }\end{array}$ & $\mathrm{n}=70$ & $\begin{array}{l}250 \mathrm{mg} \text { magnesium oxide } 6 \text { weeks } \\
\text { vs. placebo }\end{array}$ & $\begin{array}{c}-9.7 \\
\text { vs. }+1.8 \\
\text { p }<0.001\end{array}$ & $\begin{array}{c}-2.1 \text { vs. }+5.7 \\
\mathrm{p}=0.001\end{array}$ & --- \\
\hline $\begin{array}{l}\text { Magnesium and } \\
\text { Vitamin E }\end{array}$ & $\begin{array}{l}\text { Maktabi et al. } \\
2018\end{array}$ & $\begin{array}{l}\text { RCT; double } \\
\text { blind; placebo- } \\
\text { controlled }\end{array}$ & $\mathrm{n}=60$ & $\begin{array}{c}250 \mathrm{mg} / \text { day magnesium oxide plus } \\
400 \mathrm{IU} / \text { day vitamin E supplements } \\
\text { vs. placebo }\end{array}$ & $\begin{array}{c}-4.9 \text { vs. }-0.1 \\
\text { No p-value } \\
(\beta-5.20 \mathrm{mg} / \\
\mathrm{dL}(95 \% \mathrm{CI},- \\
7.88,-2.52), \mathrm{p}= \\
0.002)\end{array}$ & $\begin{array}{c}-1.7 \text { vs. }+1.5 \\
\text { No p-value } \\
(\beta-2.93(95 \% \mathrm{CI}, \\
-5.68,-0.18) \\
\mathrm{p}=0.02)\end{array}$ & -- \\
\hline Myo-inositol & $\begin{array}{l}\text { Corrado et al. } \\
2011\end{array}$ & $\mathrm{RCT}$ & $\mathrm{n}=69$ & $\begin{array}{l}\text { myoinositol (4 g daily) with folic } \\
\text { acid (400 lg daily) vs. folic acid } \\
\text { (400 lg daily) }\end{array}$ & $\begin{array}{l}-0.9 \text { vs. }-0.3 \\
p<0.05\end{array}$ & $\begin{array}{c}-12.2 \text { vs. }-7.9 \\
\mathrm{p}<0.05\end{array}$ & --- \\
\hline Probiotic & $\begin{array}{l}\text { Kijmanawat et } \\
\text { al. } 2019 .\end{array}$ & $\begin{array}{l}\text { RCT; double } \\
\text { blind; placebo- } \\
\text { controlled }\end{array}$ & $\mathrm{n}=58$ & $\begin{array}{l}\text { probiotic ( } 2 \text { bacterial strain) } 4 \\
\text { weeks vs. placebo }\end{array}$ & $\begin{array}{c}0.68 \text { vs. } 4.62 \\
p=0.03\end{array}$ & $\begin{array}{c}1.11 \text { vs. } 3.77 \\
\mathrm{p}=0.01\end{array}$ & --- \\
\hline Probiotic & $\begin{array}{l}\text { Karamali et al. } \\
2016\end{array}$ & $\begin{array}{l}\text { RCT; double } \\
\text { blind; placebo- } \\
\text { controlled }\end{array}$ & $\mathrm{n}=60$ & $\begin{array}{c}\text { probiotic ( } 3 \text { bacterial strains) } 6 \\
\text { weeks vs. placebo }\end{array}$ & $\begin{array}{c}-9.2 \text { vs. }+1.1 \\
\text { p }<0.001\end{array}$ & $\begin{array}{c}-0.8 \text { vs. }+4.5 \\
\mathrm{p}=0.01\end{array}$ & -- \\
\hline Probiotic & $\begin{array}{l}\text { Jafarnejad et al. } \\
2016\end{array}$ & $\begin{array}{l}\text { RCT; double- } \\
\text { blind; placebo }\end{array}$ & $\mathrm{n}=82$ & $\begin{array}{c}\text { probiotic ( } 3 \text { bacterial strains) } 8 \\
\text { weeks vs. placebo }\end{array}$ & $\begin{array}{c}-2.3 \text { vs. }-4.8 \\
\mathrm{p}=0.42\end{array}$ & $\begin{array}{c}-2.5 \text { vs. }+3.6 \\
p=0.04\end{array}$ & --- \\
\hline Probiotic & $\begin{array}{l}\text { Dolatkah et al. } \\
\quad 2015\end{array}$ & $\begin{array}{l}\text { RCT; double- } \\
\text { blind; placebo }\end{array}$ & $\mathrm{n}=64$ & $\begin{array}{c}\text { probiotic ( } 4 \text { bacterial strains) } 6 \\
\text { weeks vs. placebo }\end{array}$ & $\begin{array}{c}-15.3 \mathrm{vs} .- \\
\mathrm{p}=0.02\end{array}$ & $\begin{array}{c}-0.80 \text { vs. } 0.52 \\
\mathrm{p}=0.08\end{array}$ & --- \\
\hline Probiotic & $\begin{array}{l}\text { Lindsay et al. } \\
\qquad 2015\end{array}$ & $\begin{array}{l}\text { RCT; double- } \\
\text { blind; placebo }\end{array}$ & $\mathrm{n}=149$ & $\begin{array}{c}\text { probiotic (1 strain) 4-6 weeks vs. } \\
\text { placebo }\end{array}$ & $\begin{array}{c}-0.19 \text { vs. }-0.27 \\
\text { *No p-value for } \\
\text { mean change } \\
\text { however in } \\
\text { ANOVA, no } \\
\text { significant } \\
\text { difference }\end{array}$ & $\begin{array}{c}-0.84 \text { vs. }-1.03 \\
\text { *No p-value for } \\
\text { mean change } \\
\text { however in } \\
\text { ANOVA, no } \\
\text { significant } \\
\text { difference }\end{array}$ & $\begin{array}{c}17 \% \text { vs } 14 \% \\
P=.643\end{array}$ \\
\hline Synbiotic & $\begin{array}{l}\text { Nabhani et al. } \\
2017\end{array}$ & $\begin{array}{l}\text { RCT; double- } \\
\text { blind; placebo }\end{array}$ & $\mathrm{n}=90$ & $\begin{array}{l}\text { symbiotic capsule (4 strains with } \\
\text { fructooligosaccharide }(38.5 \mathrm{mg})) 6 \\
\text { weeks vs. placebo }\end{array}$ & $\begin{array}{l}\text { No significant } \\
\text { difference }\end{array}$ & $\begin{array}{l}\text { No significant } \\
\text { difference }\end{array}$ & --- \\
\hline Selenium & $\begin{array}{l}\text { Asemi et al. } \\
\quad 2015\end{array}$ & $\begin{array}{l}\text { RCT; double- } \\
\text { blind; placebo }\end{array}$ & $\mathrm{n}=70$ & $\begin{array}{l}200 \text { microgram selenium } 6 \text { weeks } \\
\text { vs. placebo }\end{array}$ & $\begin{array}{c}-10.5 \mathrm{vs} .+4.5 \\
\mathrm{p}<0.001\end{array}$ & $\begin{array}{c}-1.98 \text { vs. }+5.26 \\
\mathrm{p}=0.005\end{array}$ & --- \\
\hline Vitamin D & $\begin{array}{l}\text { Asemi et al. } \\
\quad 2013\end{array}$ & $\begin{array}{l}\text { RCT; double- } \\
\text { blind; placebo }\end{array}$ & $\mathrm{n}=54$ & $\begin{array}{c}50,000 \mathrm{IU} \text { vitamin D3 } 2 \text { times } \\
\text { (day } 0 \text { and 21) vs. placebo (day } 0 \\
\text { and } 21 \text { ) }\end{array}$ & $\begin{array}{c}-17.1 \text { vs. }-0.9 \\
p<0.001\end{array}$ & $\begin{array}{c}-3.08 \text { vs. }+1.34 \\
p=0.01\end{array}$ & -- \\
\hline Vitamin D & $\begin{array}{l}\text { Yazdchi et al. } \\
\quad 2016\end{array}$ & $\begin{array}{l}\text { RCT; double- } \\
\text { blind; placebo }\end{array}$ & $\mathrm{n}=76$ & $\begin{array}{c}50,000 \text { IU vitamin D3 } 4 \text { times (day } \\
0,14,28,42 \text { ) vs. placebo }\end{array}$ & $\begin{array}{l}-4.72 \text { vs. } 5.27 \\
\mathrm{mg} / \mathrm{dl} ; \mathrm{P}=0.01\end{array}$ & $\begin{array}{l}\text { No significant } \\
\text { difference }\end{array}$ & -- \\
\hline
\end{tabular}

intracellular functioning of endogenous insulin. In studies of patients with T2DM, myoinositol has been associated with reductions in fasting blood glucose and HbAlc levels and in patients with GDM, increased urinary excretion of inositol has been associated with blood glucose levels. In this review, one RCT was identified testing myoinositol supplementation ( $4 \mathrm{~g}$ daily) with folic acid (400 lg daily) vs. folic acid (400 lg daily) alone in 69 patients with GDM. After 8 weeks, myoinositol resulted in a significant effect on fasting insulin $(-12.2$ vs. $-7.9 ; \mathrm{p}<0.05)$, but no effect on FPG levels [72].

\section{Discussion}

\section{Supplementation}

Research on micronutrient supplementation during pregnancy, especially in patients with GDM, is extremely limited given the ethical challenges of studying this population. In this review there were 14 RCTs meeting inclusion criteria. The trials each had epidemiologic evidence or in-vitro evidence supporting their hypotheses and many were placebo-controlled. These studies assessed the impact of PUFA, MUFA, Omega-3 Fatty Acids, magnesium, selenium, probiotics, and vitamin $\mathrm{D}$. There is an overt lack of standardization and guidance for administration and dosing of supplements. With regards to probiotics, it is challenging to draw comparison between these trials given lack of standardization in probiotic supplementation related to capsule size, strain components, amount of bacteria and shelf life of probiotic supplements. Of the trials, vitamin $\mathrm{D}$, selenium and magnesium demonstrated statistically significant effects in reducing fasting plasma glucose or fasting mean insulin. PUFA, MUFA, and omega 3-fatty acids were not found to be effective in changing insulin or glucose levels. More research is needed to understand the potential impact of probiotic supplementation on glycemia. One of the probiotic trials found a profound effect on fasting plasma glucose $(-15.3 \mathrm{mg} / \mathrm{dL}$ vs. $-7.3 \mathrm{mg} / \mathrm{dL}$ ) and the other trial did not find any significant effect on 
Table 3. Summary of randomized trials assessing dietary intervention and GDM

\begin{tabular}{|c|c|c|c|c|c|c|c|}
\hline Dietary Variable & Author; Year & Design & n & Independent variable & $\begin{array}{l}\text { Mean change in } \\
\text { FPG }(\mathbf{m g} / \mathbf{d L})\end{array}$ & $\begin{array}{l}\text { Mean change in } \\
\text { serum insulin } \\
(\mathrm{mIU} / \mathrm{mL})\end{array}$ & $\begin{array}{l}\text { Initiation of } \\
\text { Insulin (\%) }\end{array}$ \\
\hline $\begin{array}{l}\text { PUFA (Sunflower oil- } \\
\text { rich diet) }\end{array}$ & Wang et al. 2015 & $\begin{array}{l}\text { RCT; not } \\
\text { blinded }\end{array}$ & $\mathrm{n}=84$ & $\begin{array}{l}\text { High PUFA vs. Low PUFA - } \\
\text { Isocaloric }\end{array}$ & No difference & No difference & --- \\
\hline MUFA & $\begin{array}{l}\text { FF Lauszus et al. } \\
\quad 2002\end{array}$ & $\begin{array}{l}\text { RCT; not } \\
\text { blinded }\end{array}$ & $\mathrm{n}=27$ & $\begin{array}{c}\text { High MUFA for } 5 \text { weeks vs Low } \\
\text { MUFA }\end{array}$ & No difference & No difference & --- \\
\hline DASH & Asemi et al. 2014 & $\begin{array}{l}\text { RCT; single } \\
\quad \text { blind; }\end{array}$ & $\mathrm{n}=32$ & $\begin{array}{l}\text { DASH for } 4 \text { weeks (vegetables, } \\
\text { whole grains, etc.) vs control }\end{array}$ & $\begin{array}{c}-7.62 \text { vs. } 3.68 \\
p=0.02\end{array}$ & $\begin{array}{c}-2.62 \text { vs. } 4.32 \\
p=0.03\end{array}$ & --- \\
\hline DASH & Asemi et al. 2013 & $\begin{array}{l}\text { RCT; single } \\
\text { blind }\end{array}$ & $\mathrm{n}=53$ & DASH for 4 weeks vs control & --- & --- & $\begin{array}{c}23 \% \text { vs. } 73 \% \\
\text { p }<0.0001\end{array}$ \\
\hline Low Calorie & Rae et al. 2000 & $\begin{array}{l}\text { RCT; double- } \\
\text { blinded }\end{array}$ & $\mathrm{n}=120$ & $\begin{array}{l}30 \% \text { caloric reduction for } 12 \text { weeks } \\
\text { vs. control }\end{array}$ & No difference & --- & No difference \\
\hline $\begin{array}{l}\text { High complex carb/ } \\
\text { Low Fat }\end{array}$ & $\begin{array}{l}\text { Hernandez et al. } \\
2016\end{array}$ & $\begin{array}{l}\text { RCT; not } \\
\text { blinded }\end{array}$ & $\mathrm{n}=12$ & $\begin{array}{l}\text { High complex carb (low fat) vs. } \\
\text { Low Carb (high fat) - Isocaloric }\end{array}$ & $\begin{array}{l}\text { "Decreased with } \\
\text { intervention" (mean \# not } \\
\text { provided) }\end{array}$ & No difference & --- \\
\hline $\begin{array}{l}\text { High Complex Carb/ } \\
\text { Low Fat }\end{array}$ & $\begin{array}{l}\text { Hernandez et al. } \\
\quad 2014\end{array}$ & $\begin{array}{c}\text { RCT; cross-over } \\
\text { design }\end{array}$ & $\mathrm{n}=16$ & $\begin{array}{l}\text { High complex carb (low fat) vs. } \\
\text { Low Carb (high fat) - Isocaloric }\end{array}$ & No difference & No difference & ---- \\
\hline $\begin{array}{l}\text { Low Carb vs. High } \\
\text { Carb }\end{array}$ & $\begin{array}{c}\text { Morena-Castilla et } \\
\text { al. } 2013\end{array}$ & $\begin{array}{l}\text { RCT; not } \\
\text { blinded }\end{array}$ & $\mathrm{n}=152$ & $\begin{array}{l}40 \% \text { CHO diet vs. } 55 \% \text { CHO diet } \\
\text {-- isocaloric and similar amount } \\
\text { protein }\end{array}$ & --- & --- & No difference \\
\hline $\begin{array}{l}\text { Low Carb vs. High } \\
\text { Carb }\end{array}$ & Cypryk et al. 2007 & $\begin{array}{l}\text { RCT; not } \\
\text { blinded }\end{array}$ & $\mathrm{n}=30$ & $\begin{array}{c}\text { Low Carb ( } 45 \% \text { of kcals }) \text { vs. High } \\
\text { Carb }(\sim 65 \% \text { of kcals })\end{array}$ & No difference & No difference & --- \\
\hline $\begin{array}{l}\text { Low Glycemic Index } \\
\text { Diet }\end{array}$ & Louie et al. 2011 & $\begin{array}{l}\text { RCT; not } \\
\text { blinded }\end{array}$ & $\mathrm{n}=99$ & $\begin{array}{c}\text { Low GI (Mean GI: 47) vs. } \\
\text { Moderate GI with fiber (Mean } \\
\text { GI: } 53 \text { ) } \\
\text { Protein, carb and fat controlled }\end{array}$ & --- & No difference & No difference \\
\hline Low Glycemic Index & Hu et al. 2014 & $\begin{array}{l}\text { RCT; not } \\
\text { blinded }\end{array}$ & $\mathrm{n}=140$ & $\begin{array}{c}\text { Low-GI (brown rice based) vs. } \\
\text { Diabetic Control (white rice based) } \\
\text { - Isocaloric }\end{array}$ & $\begin{array}{c}-3.7 \% \text { ( } \% \text { changes from } \\
\text { baseline) vs. }-1.2 \% \\
\text { p }<0.05\end{array}$ & --- & --- \\
\hline Low Glycemic Index & Moses et al. 2009 & $\begin{array}{l}\text { RCT; not } \\
\text { blinded }\end{array}$ & $\mathrm{n}=63$ & $\begin{array}{l}\text { Low GI foods (avoid white bread, } \\
\text { white potatoes, baked goods) } \\
\text { vs. High fiber/Low Sugar foods } \\
\text { (Potatoes; whole wheat bread } \\
\text { encouraged) }\end{array}$ & --- & --- & $\begin{array}{c}29 \% \text { vs. } 59 \% \\
\quad p=0.023\end{array}$ \\
\hline $\begin{array}{l}\text { Low Glycemic Index } \\
\text { with Fiber }(15 \mathrm{~g} / \mathrm{d})\end{array}$ & Afaghi et al. 2013 & $\begin{array}{l}\text { RCT; not } \\
\text { blinded }\end{array}$ & $\mathrm{n}=36$ & $\begin{array}{c}\text { Low GI with } 15 \mathrm{~g} \text { fiber } 2 \text { weeks vs. } \\
\text { Low GI }\end{array}$ & No difference & --- & $\begin{array}{l}38.9 \% \text { vs. } 76.9 \% \\
\text { *no p-value }\end{array}$ \\
\hline Low Glycemic Index & Ma et al. 2014 & $\begin{array}{l}\text { RCT; not } \\
\text { blinded }\end{array}$ & $\mathrm{n}=95$ & $\begin{array}{l}\text { Low GI 10-12 weeks (with re- } \\
\text { education every } 2 \text { weeks) vs. } \\
\text { Control GDM }\end{array}$ & $\begin{array}{c}-5.9 \text { vs. }-.36 \\
\mathrm{p}<0.01\end{array}$ & --- & --- \\
\hline Low Glycemic Index & Grant et al. 2011 & $\begin{array}{l}\text { RCT; not } \\
\text { blinded }\end{array}$ & $\mathrm{n}=47$ & $\begin{array}{l}\text { Low GI (Mean GI=49) vs. Control } \\
\qquad(\mathrm{GI}=58)\end{array}$ & No difference & --- & --- \\
\hline
\end{tabular}

glucose or insulin, however it is difficult to draw conclusions given the difference in amount and quantity of bacterial strains tested.

\section{Diet}

Overall, high quality, large-scale or multi-site randomized controlled-trials evaluating the effects of diet and macronutrient variation on glycemia in samples of females with GDM are limited. In this systematic literature review, we found 15 randomized-controlled trials meeting inclusion criteria which evaluated the impact of diet on either mean change fasting blood glucose or mean insulin change (Table 3). Although leading medical organizations do underscore the importance of lifestyle modification as first line therapy for GDM to prevent adverse perinatal outcomes (Table 2), in general they do not make specific dietary recommendations beyond typical prenatal recommendations. In our review, we found 15 RCTs assessing the impact of five diets 1) high complex carbohydrate (low fat) diet, 2) low GI diet, 3) low carbohydrate diet, 4) low calorie diet, and the 5) DASH diet. The low GI diet was the most researched as this diet had 6 RCTs whereas the other diets had only 1-2 RCTs. The low GI diet also had the most evidence supporting its effect on mean fasting plasma glucose change and mean insulin change with the greatest magnitude of effect. Moreover, the trials were controlled isocaloric studies meaning both the intervention and control group were consuming the same amount of calories and likely the same amount of carbohydrate and fat. The difference in outcome is likely attributable to the prioritization of low glycemic carbohydrates like yogurt, nuts, and legumes which are evidenced to have slow or minimal effect on blood glucose. Interestingly, the glycemic index diet is not always intuitive. Both brown rice and white rice have similar glycemic scores even though one might assume that the added fiber in brown rice would blunt the rise in postprandial glucose [62].

In addition, two of the trials evaluating the effect of low GI diet on GDM reported statistically significant differences in proportion of patients having to commence insulin injectable therapy. This suggests that the low GI index diet improves glycemic control such that fewer patients have to start insulin therapy. This is helpful for patients 
because insulin injections are expensive and often not well-tolerated nor accepted by pregnant women. In addition to the low GI diet, the DASH diet which is inherently low-glycemic and tends to be lower in calories was found in one trial to improve mean fasting glucose, fasting insulin and in another to reduce the proportion of patients requiring insulin injections. The other trials assessing the low calorie diet, the high complex carbohydrate diet and the low carbohydrate diet did not demonstrate significant effect on glycemia in patients with GDM. It is worth mentioning that the low carbohydrate diet study was very small, relied on self-report, and the carbohydrate percentage between intervention and control differed by $15 \%$. It may be that DASH and low GI diets work well because they tend to be "lower carbohydrate." Some researchers have also argued that the requirement to have at least $175 \mathrm{~g}$ of carbohydrate per day during pregnancy is not biologically necessary and is not evidence-based [64]. In the future, it may make sense to further evaluate varying amounts of carbohydrate within the low GI index parameters to identify optimal amount of carbohydrate for euglycemia.

Although the trials are generally smaller samples and short-term, they do lend support for the low glycemic index diet as a way to improve glycemia in women with GDM and ultimately reduce the likelihood of poor neonatal outcomes and adverse childhood outcomes [65-70].

\section{Limitations}

As listed in Table 3, there are many threats to internal and external validity impacting our ability to draw conclusions. Most studies were conducted on small $(n<100)$, homogenous samples limiting generalizability. Many of the samples excluded patients with more severe GDM or patients with preexisting risk factors. In addition, most trials were short-term (4-6 weeks), reliant on self-report and unblinded.

\section{Conclusions}

Trends suggest that lower glycemic index diet, lower carbohydrate diets and diets with lean protein and high fruits and vegetables may be effective in reducing hyperglycemia in GDM. As for supplementation, more research is needed. Supplements are a simple, and generally low-cost intervention to manage glycemia in patients with with GDM and may be more acceptable to patients. From this literature review, it appears that vitamin D, selenium, and magnesium may signal an effect on plasma glucose and insulin. However, studies were scarce so more research is needed to confirm these trends. It is valuable to mention that other factors may play a role in lifestyle management of gestational diabetes such as routine exercise (the general recommendation is 150 minutes per week) and frequent or intensive counseling with a dietitian or healthcare practitioner, however the focus of this paper is dietary and supplement-based prescriptions. Intensive counseling and exercise routines were not components of the supplementation trials and if these were included in the diet-based trials, were generally controlled for by equal provision between the intervention and control group.

At present, there is more evidence supporting the glycemic index diet than other dietary interventions their. This aligns with the AAFP's recent adoption of glycemic index into their dietary recommendations. Many other professional organizations have not updated their recommendations and still cling to the general recommendation for pregnancy of at least 175 grams of carbohydrate per day to meet the needs of the growing fetus and prevent ketosis. We need more randomized trials evaluating differing amounts of carbohydrate within glycemic index parameters in order to gain consensus within the field, crystallize dietary guidelines, and ultimately prevent the adverse perinatal and neonatal impacts of GDM.

\section{References}

1. Moses RG, Cheung NW (2009) Point: universal screening for gestational diabetes mellitus. Am Diabetes Assoc.

2. Ben-Haroush A, Yogev Y, Hod M (2004) Epidemiology of gestational diabetes mellitus and its association with Type 2 diabetes. Diabetic Medicine 21: 103-113.

3. Di Cianni G, Miccoli R, Volpe L, Lencioni C, Del Prato S (2003) Intermediate metabolism in normal pregnancy and in gestational diabetes. Diabetes/Metabolism Research and Reviews 19: 259-270.

4. Sonagra AD, Biradar SM, Dattatreya K, DS JM (2014) Normal pregnancy-a state of insulin resistance. Journal of clinical and diagnostic research: JCDR 8: CC01.

5. DeSisto CL, Kim SY, Sharma AJ (2014) Peer reviewed: Prevalence estimates of gestational diabetes mellitus in the United States, pregnancy risk assessment monitoring system (prams), 2007-2010. Preventing Chronic Disease.

6. Nguyen CL, Pham NM, Binns CW, Duong DV, Lee AH (2018) Prevalence of gestational diabetes mellitus in eastern and southeastern Asia: A systematic review and meta-analysis. Journal of diabetes research.

7. Luke B, Brown MB (2007) Elevated risks of pregnancy complications and adverse outcomes with increasing maternal age. Human Reproduction 22: 1264-1272.

8. Moyer VA (2014) Screening for gestational diabetes mellitus: US preventive services task force recommendation statement. Annals of Internal Medicine 160: 414-420.

9. Carpenter MW, Coustan DR (1982) Criteria for screening tests for gestational diabetes American Journal of Obstetrics \& Gynecology 144: 768-773.

10. Gluckman PD, Cutfield W, Hofman P, Hanson MA (2005) The fetal, neonatal, and infant environments-the long-term consequences for disease risk. Early Human Development 81: 51-59.

11. Nold JL, Georgieff MK (2004) Infants of diabetic mothers. Pediatric Clinics 51: 619637.

12. Goldman AS, Baker L, Piddington R, Marx B, Herold R, et al. (1985) Hyperglycemiainduced teratogenesis is mediated by a functional deficiency of arachidonic acid. Proceedings of the National Academy of Sciences 82: 8227-8231.

13. Kim C, Newton KM, Knopp RH (2002) Gestational diabetes and the incidence of type 2 diabetes: a systematic review. Diabetes Care 25: 1862-1868.

14. Group HSCR (2008) Hyperglycemia and adverse pregnancy outcomes. New England Journal of Medicine 358: 1991-2002.

15. Moreno-Castilla C, Mauricio D, Hernandez M (2016) Role of medical nutrition therapy in the management of gestational diabetes mellitus. Current Diabetes Reports 16: 22.

16. Moses RG, Barker M, Winter M, Petocz P, Brand-Miller JC (2009) Can a low-glycemic index diet reduces the need for insulin in gestational diabetes mellitus? A randomized trial. Diabetes Care 32: 996-1000.

17. Mellitus GD. ACOG PRACTICE BULLETIN.

18. Association AD (2018) Lifestyle management: Standards of medical care in diabetes-2018. Diabetes Care 41: S38-S50.

19. Garrison A (2015) Screening, diagnosis, and management of gestational diabetes mellitus. Am Fam Physician 91: 460-467.

20. Colagiuri S, Cull CA, Holman RR (2002) Are lower fasting plasma glucose levels at diagnosis of type 2 diabetes associated with improved outcomes? UK prospective diabetes study 61. Diabetes Care 25: 1410-1417.

21. Pyörälä K (1979) Relationship of glucose tolerance and plasma insulin to the incidence of coronary heart disease: results from two population studies in Finland. Diabetes Care 2: 131-141.

22. Retnakaran R, Qi Y, Sermer M, Connelly PW, Hanley AJ, et al. (2009) The antepartum glucose values that predict neonatal macrosomia differ from those that predict postpartum prediabetes or diabetes: implications for the diagnostic criteria for gestational diabetes. The Journal of Clinical Endocrinology \& Metabolism 94: 840845 .

23. Hashimoto K, Koga M (2015) Indicators of glycemic control in patients with gestational diabetes mellitus and pregnant women with diabetes mellitus. World Journal of Diabetes 6: 1045.

24. Gross LS, Li L, Ford ES, Liu S (2004) Increased consumption of refined carbohydrates and the epidemic of type 2 diabetes in the United States: an ecologic assessment. The American Journal of Clinical Nutrition 79: 774-779. 
25. Major CA, Henry MJ, De Veciana M, Morgan MA (1998) The effects of carbohydrate restriction in patients with diet-controlled gestational diabetes. Obstetrics \& Gynecology 91: 600-604.

26. Moreno-Castilla C, Hernandez M, Bergua M, Alvarez MC, Arce MA, et al. (2013) Low-carbohydrate diet for the treatment of gestational diabetes mellitus: a randomized controlled trial. Diabetes Care 36: 2233-2238. [Crossref]

27. Jenkins D, Wolever T, Collier GR, Ocana A, Rao AV, et al. (1987) Metabolic effects of a low-glycemic-index diet. Am J Clin Nutr 46: 968-975. [Crossref]

28. Augustin LS, Kendall CW, Jenkins DJ, Willett WC, Astrup A, et al. (2015) Glycemic index, glycemic load and glycemic response: An international scientific consensus summit from the international carbohydrate quality consortium (ICQC). Nutrition, Metabolism and Cardiovascular Diseases 25: 795-815. [Crossref]

29. Louie JCY, Markovic TP, Perera N, Foote D, Petocz P, et al. (2011) A randomized controlled trial investigating the effects of a low-glycemic index diet on pregnancy outcomes in gestational diabetes mellitus. Diabetes Care 34: 2341-2346. [Crossref]

30. Carolan-Olah M, Duarte-Gardea M, Lechuga J (2017) A systematic review of interventions for Hispanic women with or at risk of Gestational diabetes mellitus (GDM). Sexual \& Reproductive Healthcare 13: 14-22.

31. Hu ZG, Tan RS, Jin D, Li W, Zhou XY (2014) A low glycemic index staple diet reduces postprandial glucose values in Asian women with gestational diabetes mellitus. Journal of Investigative Medicine 62: 975-979.

32. Moses RG, Barker M, Winter M, Petocz P, Brand-Miller JC (2009) Can a low-glycemic index diet reduces the need for insulin in gestational diabetes mellitus? A randomized trial. Diabetes Care 32: 996-1000.

33. Ma WJ, Huang ZH, Huang BX, Qi BH, Zhang YJ, et al. (2015) Intensive lowglycaemic-load dietary intervention for the management of glycaemia and serum lipids among women with gestational diabetes: a randomized control trial. Public Health Nutr 18: 1506-1513. [Crossref]

34. Grant SM, Wolever TM, O'Connor DL, Nisenbaum R, Josse RG (2011) Effect of a low glycaemic index diet on blood glucose in women with gestational hyperglycaemia. Diabetes Research and Clinical Practice 91: 15-22.

35. Viana LV, Gross JL, Azevedo MJ (2014) Dietary intervention in patients with gestational diabetes mellitus: a systematic review and meta-analysis of randomized clinical trials on maternal and newborn outcomes. Diabetes Care 37: 3345-3355.

36. Afaghi A, Ghanei L, Ziaee A (2013) Effect of low glycemic load diet with and withou wheat bran on glucose control in gestational diabetes mellitus: A randomized trial. Indian Journal of Endocrinology and Metabolism 17: 689

37. Risérus U, Willett WC, Hu FB (2009) Dietary fats and prevention of type 2 diabetes. Progress in Lipid Research 48: 44-51.

38. Kim Y, Tomohiro T, Iwashita S, Tokuyama K, Suzuki M (1994) Effect of high-fat die on gene expression of GLUT4 and insulin receptor in soleus muscle. Biochemical and Biophysical Research Communications 202: 519-526.

39. Chen X, Scholl TO, Leskiw M, Savaille J, Stein TP (2010) Differences in materna circulating fatty acid composition and dietary fat intake in women with gestational diabetes mellitus or mild gestational hyperglycemia. Diabetes Care 33: 2049-2054.

40. Wang HY, Jiang HY, Yang LP, Zhang M (2015) Impacts of dietary fat changes on pregnant women with gestational diabetes mellitus: a randomized controlled study. Asia Pacific Journal of Clinical Nutrition 24: 58-64.

41. Vessby B (2003) Dietary fat, fatty acid composition in plasma and the metabolic syndrome. Current Opinion in Lipidology 14: 15-19.

42. Qian F, Korat AA, Malik V, Hu FB (2016) Metabolic effects of monounsaturated fatty acid-enriched diets compared with carbohydrate or polyunsaturated fatty acidenriched diets in patients with type 2 diabetes: a systematic review and meta-analysis of randomized controlled trials. Diabetes Care 39: 1448-1457.

43. Lauszus F, Rasmussen O, Henriksen JE, Klebe JG, Jensen L, et al. (2002) Effect of a high monounsaturated fatty acid diet on blood pressure and glucose metabolism in women with gestational diabetes mellitus. Eur J Clin Nutr 55: 436. [Crossref]

44. Harnden K, Frayn K, Hodson L (2010) Dietary approaches to stop hypertension (DASH) diet: applicability and acceptability to a UK population. J Hum Nutr Diet 23: 3-10. [Crossref]

45. Azadbakht L, Mirmiran P, Esmaillzadeh A, Azizi T, Azizi F (2005) Beneficial effects of a dietary approaches to stop hypertension eating plan on features of the metabolic syndrome. Diabetes Care 28: 2823-2831.
46. Asemi Z, Samimi M, Tabassi Z, Esmaillzadeh A (2014) The effect of DASH diet on pregnancy outcomes in gestational diabetes: a randomized controlled clinical trial. Eur J Clin Nutr 68: 490. [Crossref]

47. Asemi Z, Samimi M, Tabassi Z, Sabihi S, Esmaillzadeh A (2013) A randomized controlled clinical trial investigating the effect of DASH diet on insulin resistance, inflammation, and oxidative stress in gestational diabetes. Nutrition 29: 619-624.

48. Hernandez TL, Van Pelt RE, Anderson MA, Reece MS, Reynolds RM, et al (2016) Women with gestational diabetes mellitus randomized to a higher-complex carbohydrate/low-fat diet manifest lower adipose tissue insulin resistance, inflammation, glucose, and free fatty acids: a pilot study. Diabetes Care 39: 39-42. [Crossref]

49. Hernandez TL, Van Pelt RE, Anderson MA, Daniels LJ, West NA, et al. (2014) A higher-complex carbohydrate diet in gestational diabetes mellitus achieves glucose targets and lowers postprandial lipids: a randomized crossover study. Diabetes Care 37: 1254-1262. [Crossref]

50. Wehmeier K, Beers A, Haas MJ, Wong NC, Steinmeyer A, et al. (2005) Inhibition of apolipoprotein AI gene expression by 1, 25-dihydroxyvitamin D3. Biochim Biophys Acta 1737: 16-26.

51. Lee CJ, Iyer G, Liu Y, Kalyani RR, Bamba N, et al. (2017) The effect of vitamin D supplementation on glucose metabolism in type 2 diabetes mellitus: A systematic review and meta-analysis of intervention studies. J Diabetes Complications 31: 11151126. [Crossref]

52. Asemi Z, Karamali M, Esmaillzadeh A (2014) Effects of calcium-vitamin D cosupplementation on glycaemic control, inflammation and oxidative stress in gestational diabetes: a randomised placebo-controlled trial. Diabetologia 57: 1798-1806. [Crossref]

53. von Hurst PR, Stonehouse W, Coad J (2010) Vitamin D supplementation reduces insulin resistance in South Asian women living in New Zealand who are insulin resistant and vitamin D deficient-a randomised, placebo-controlled trial. Br J Nutr 103: 549-555. [Crossref]

54. Yazdchi R, Gargari BP, Asghari-Jafarabadi M, Sahhaf F (2016) Effects of vitamin $\mathrm{D}$ supplementation on metabolic indices and hs-CRP levels in gestational diabetes mellitus patients: a randomized, double-blinded, placebo-controlled clinical trial. Nutr Res Pract 10: 328-335. [Crossref]

55. Ostadrahimi A, Mohammad-Alizadeh S, Mirgafourvand M, Yaghoubi S, Shahrisa E, et al. (2016) Effects of fish oil supplementation on gestational diabetes mellitus (GDM): a systematic review. Iranian Red Crescent Medical Journal 18.

56. Pifferi F, Dorieux O, Castellano CA, Croteau E, Masson M, et al. (2015) Long-chain n-3 PUFAs from fish oil enhance resting state brain glucose utilization and reduce anxiety in an adult nonhuman primate, the grey mouse lemur. J Lipid Res 56: 1511-1518.

57. Samimi M, Jamilian M, Asemi Z, Esmaillzadeh A (2015) Effects of omega-3 fatty acid supplementation on insulin metabolism and lipid profiles in gestational diabetes: randomized, double-blind, placebo-controlled trial. Clin Nutr 34: 388-393.

58. Askari G, Iraj B, Salehi-Abargouei A, Fallah AA, Jafari T (2015) The association between serum selenium and gestational diabetes mellitus: a systematic review and meta-analysis. Journal of Trace Elements in Medicine and Biology 29: 195-201.

59. Asemi Z, Jamilian M, Mesdaghinia E, Esmaillzadeh A (2015) Effects of selenium supplementation on glucose homeostasis, inflammation, and oxidative stress in gestational diabetes: Randomized, double-blind, placebo-controlled trial. Nutrition 31: $1235-1242$.

60. Vlassara H, Uribarri J (2014) Advanced glycation end products (AGE) and diabetes: cause, effect, or both? Current Diabetes Reports 14: 453.

61. Taylor BL, Woodfall GE, Sheedy KE, O'Riley ML, Rainbow KA, et al. (2017) Effect of probiotics on metabolic outcomes in pregnant women with gestational diabetes: a systematic review and meta-analysis of randomized controlled trials. Nutrients 9: 461. [Crossref]

62. Karamali M, Dadkhah F, Sadrkhanlou M, Jamilian M, Ahmadi S, et al. (2016) Effects of probiotic supplementation on glycaemic control and lipid profiles in gestational diabetes: a randomized, double-blind, placebo-controlled trial. Diabetes Metab 42 : 234-241.

63. Dolatkhah N, Hajifaraji M, Abbasalizadeh F, Aghamohammadzadeh N, Mehrabi Y, et al. (2015) Is there a value for probiotic supplements in gestational diabetes mellitus? A randomized clinical trial. Journal of Health, Population and Nutrition 33: 25.

64. Lindsay KL, Brennan L, Kennelly MA, Maguire OC, Smith T, et al. (2015) Impact of probiotics in women with gestational diabetes mellitus on metabolic health: a randomized controlled trial. Am J Obstet Gynecol 212: 496. [Crossref] 
65. Jafarnejad S, Saremi S, Jafarnejad F, Arab A (2016) Effects of a multispecies probiotic mixture on glycemic control and inflammatory status in women with gestational diabetes: a randomized controlled clinical trial. J Nutr Metab.

66. Barbagallo M, Dominguez LJ (2015) Magnesium and type 2 diabetes. World Journal of Diabetes 6: 1152 .

67. Bardicef M, Bardicef O, Sorokin Y, Altura BM, Altura BT, et al. (1995) Extracellular and intracellular magnesium depletion in pregnancy and gestational diabetes. $\mathrm{Am} \mathrm{J}$ Obstet Gynecol 172: 1009-1013. [Crossref]

68. Asemi Z, Karamali M, Jamilian M, Foroozanfard F, Bahmani F, et al. (2015) Magnesium supplementation affects metabolic status and pregnancy outcomes in gestational diabetes: a randomized, double-blind, placebo-controlled trial. Am J Clin Nutr 102: 222-229. [Crossref]

69. Atkinson FS, Foster-Powell K, Brand-Miller JC (2008) International tables of glycemic index and glycemic load values: 2008. Diabetes Care 31: 2281-2283. [Crossref]

70. Hone J, Jovanovic L (2010) Approach to the patient with diabetes during pregnancy. $J$ Clin Endocrinol Metab 95: 3578-3585. [Crossref]
71. Rae A, Bond D, Evans S, North F, Roberman B, et al. (2000) A randomised controlled trial of dietary energy restriction in the management of obese women with gestational diabetes. Australian and New Zealand Journal of Obstetrics and Gynaecology 40: 416422. [Crossref]

72. Corrado F, D'Anna R, Di Vieste G, Giordano D, Pintaudi B, et al. (2011) The effect of myoinositol supplementation on insulin resistance in patients with gestational diabetes. Diabetic Medicine 28: 972-975.

73. Kijmanawat A, Panburana P, Reutrakul S, Tangshewinsirikul C (2019)Effects of probiotic supplements on insulin resistance in gestational diabetes mellitus: A doubleblind randomized controlled trial. J Diabetes Investig10: 163-170.

74. Saleh L, Schrier NL, Bruins MJ, Steegers EAP, van den Meiracker AH, et al. (2018) Effect of oral protein hydrolysate on glucose control in patients with gestational diabetes. Clinical Nutrition 37: 878-883.

75. 75. Maktabi M, Jamilian M, Amirani E, Chamani M, Asemi Z (2018) The effects of magnesium and vitamin $\mathrm{E}$ co-supplementation on parameters of glucose homeostasis and lipid profiles in patients with gestational diabetes. Lipids Health Dis 17: 163.

Copyright: $(02020$ Viglione C. This is an open-access article distributed under the terms of the Creative Commons Attribution License, which permits unrestricted use, distribution, and reproduction in any medium, provided the original author and source are credited. 